\title{
Quality of Life in Chronic Kidney Disease Patients Using a Synbiotic Dietary Supplement: A Survey
}

\author{
Ranganathan Natarajan', Pari Ranganathan', Henry D'Silva', Usha Vyas', Bohdan \\ Pechenyak $^{2}$, Alan Weinberg ${ }^{3}$ \\ ${ }^{I}$ Kibow Biotech, Inc., Newtown Square, PA, USA \\ ${ }^{2}$ Temple University School of Medicine, Philadelphia, PA, USA \\ ${ }^{3}$ Mount Sinai School of Medicine, New York, NY, USA
}

\begin{abstract}
Background: A synbiotic dietary supplement Renadyl ${ }^{\mathrm{TM}}$, which is being used by over 3000 CKD customers, was studied in a customer survey. The focus was health status and Quality of Life (QoL). Methods: Survey questionnaires were sent to 951 current repeat customers of Kibow Biotech Inc. Of those, 117 were excluded due to mailing errors and other reasons. The final sample size was 834. Results were tabulated and analysed using Statistical Analysis Software (SAS) V9.2 and Microsoft MS Excel. Results: A total of 168 responses were received (20\% response rate, $42 \%$ female, $47 \%$ male, ages $12-98$ years). A majority (85\%) were over 51 years of age, in stage III or IV of kidney disease $(58 \%)$ with at least one comorbid condition (77\%), and almost half $(48 \%)$ were retired. A majority $(61 \%)$ reported experiencing at least some or even great improvement since they started taking Renadyl ${ }^{\mathrm{TM}}$. Statistical analysis indicated a significant difference $(\mathrm{p}<0.0001)$ in distributions in quality of life when comparing responses before and after taking Renadyl. Multivariate analysis indicated that the duration of Renadyl TM administration $(p<0.0001)$, employment $(p<0.012)$, comorbidity $(p<0.012)$, and Glomerular Filtration Rate (GFR) ( $\mathrm{p}<0.0015)$ were significant factors influencing the reported quality of life. Even the disabled respondents all reported significant improvement. Conclusions: Renadyl ${ }^{\mathrm{TM}}$ appears to provide at least some benefit in all stages of CKD and with a variety of comorbid conditions. It does not interfere with any other medical treatments, including dialysis. It appears to have a stabilizing effect on the overall health status and quality of life, maintaining or improving kidney health in particular. These findings reinforce the results of our 2013 survey and highlight the possible potential of modulating the gut microbiome with specifically chosen combination of probiotic strains and prebiotics. Further adequately powered studies that could establish a clearer correlation between Renadyl ${ }^{\mathrm{TM}}$ and its impact on GFR are warranted.
\end{abstract}

Keywords: Chronic Kidney Disease, Gut Microbiome, Probiotics, Renadyl ${ }^{\mathrm{TM}}$, Survey

\section{INTRODUCTION}

Kidney disease is the ninth leading cause of death in the U.S. [1], with over 660,000 End-Stage Renal Disease (ESRD) patients (most of them on dialysis) and more than 30 million (13.6\% of adult U.S. population) in earlier stages of Chronic Kidney Disease (CKD) [2]. Over 2 million people receive either dialysis or a kidney transplant worldwide, however this may be represent just $10 \%$ of the population who need it [3]. As the population ages, the epidemiology shifts to chronic metabolic diseases, such as obesity, diabetes and high blood pressure, all contributing factors to kidney disease. The annual cost of ESRD, according to U.S. Renal Data Systems, is $\$ 50$ billion, while that of CKD, in Medicare patients alone, is another $\$ 45.5$ billion. It is likely that people in the U.S. and globally will have a major health crisis in kidney disease in the coming years. A recent review and meta-analysis has shown that CKD has a high global prevalence between 11 to $13 \%$, with the majority being in stage 3 of CKD [4].

The role of the digestive system [5], as well as inflammation [6] and oxidative stress [7, 8] in kidney disease progression has been emphasized by researchers in the past decade. Current data have highlighted an integrated and perhaps a causal relationship between the observed clinical outcomes and the role of an activated immune system in uremia [9]. In recent years, Kidney International reviewed the role of microbial imbalance (dysbiosis) in CKD and the extent to which the gut microbial population might play a permissive role in the generation or assist in the degradation (perhaps even both) of many of the uremic toxins $[9,10]$. 
The use of probiotics and prebiotics in health and illness has expanded rapidly. A simple search of the National Institute of Health (NIH) clinicaltrials.gov registry for "probiotics" brought up 810 clinical studies [11]. Though we have accumulated some scientific evidence to substantiate their use in conditions and illnesses such as chronic kidney disease (CKD), we must continue to study their therapeutic potential whenever mechanisms that explain illnesses and adverse conditions provide the scientific basis for use. As the safety and health benefits are established, it is reasonable to anticipate that probiotic bacteria will be incorporated into a growing number of clinical regimens, either on their own or as an adjunct/part of a combined treatment, including kidney disease.

Over the past 15 years, the potential utilization of oral sorbents and probiotics as complementary strategy for CKD has continuously been explored, both in vitro and in vivo [12], in rat and mini pig animal trials [13,14], in veterinary trials [15], and in human clinical trials with CKD stages 3 and 4 patients [16-19]. The first patented and proprietary probiotic dietary supplement formulation to maintain kidney health was developed in 2009 - KibowBiotics ${ }^{\circledR}$ (now Renadyl ${ }^{\mathrm{TM}}$, Kibow Biotech, Inc., Newtown Square, PA, USA), containing S.thermophilus KB 19, L.acidophilus KB 27 and B.longum KB 31 strains, with a total of 45 billion colony forming units (CFU) per capsule. It uses "enteric toxin removal technology" to specifically target and reduce several uremic toxins that diffuse from circulating blood across the bowel and contribute to CKD. Throughout the entire R\&D process, Renady ${ }^{\mathrm{TM}}$ has shown the ability to utilize various nitrogenous uremic toxins as nutrients for growth of the beneficial gut microbial population, thus keeping the toxins from accumulating to highly toxic levels in patients with CKD. Unlike many untested probiotic supplements available on the market, Renadyl $^{\mathrm{TM}}$ has the advantage of having proven scientific validity [12-19]. The results of the randomized human clinical study in ESRD patients (CKD stage 5) on hemodialysis clearly indicated the safety of usage of Renadyl with reduction in the gut derived uremic toxins like indoxyl glucuronide [20]. Due to limited patient sample size, the efficacy of Renadyl in patients undergoing hemodialysis could not be adequately assessed [20].

Renadyl $^{\mathrm{TM}}$ has been available for purchase since 2010 via the company's own online store only. Since then, a solid base of long-term repeat customers has been established and continues to grow. Given the overwhelmingly positive feedback from these customers, a need to systematize this anecdotal evidence became apparent, and the first customer satisfaction survey was conducted in the fall of 2013 [21]. The aim of that survey study was to collect information about the quality of life and health status of the customers that had been using Renadyl ${ }^{\mathrm{TM}}$. Subsequently, in the winter of 2015, an abbreviated customer survey was conducted again, to collect basic information on the quality of life and health status of Renadyl ${ }^{\mathrm{TM}}$ customers and to compare the results to those of the previous study. The results of this 2015 survey are presented below.

\section{METHODS}

A survey questionnaire similar to the one we used for our previous survey was designed, using the combined expertise gleaned both from experience in medical/healthcare professions, including public health, and from sociological training and social science research methods. To ensure the internal validity of the questionnaire, internal controls were used, such as question rephrasing and repetition. During the week of November 16th, 2015, almost one thousand surveys were mailed out to all of the current repeat customers $(n=951)$. As an incentive to complete the survey, all respondents were offered a $25 \%$ discount on their next order of Renadyl ${ }^{\mathrm{TM}}$. We indicated November 30, 2015 as the preferred response date, but we continued collecting the incoming surveys until December 31 . In addition, to increase the response rate, an e-mail solicitation to fill out the survey was sent out on December 15, using our database and e-mail service with Constant Contact.

Out of 951 questionnaires mailed, 117 were excluded from the sample for various reasons: returned to sender by the U.S. Postal Service ( $\mathrm{n}=70$, mostly due to insufficient address information, inability to forward the mailing, or other mailing issues), the addressees refused to fill out the survey $(n=5)$ or returned an incomplete survey $(n=22)$, had passed away $(n=7)$, had given the product to their pets with kidney issues $(n=7)$, or simply mailed in the filled-out questionnaire significantly later than we had originally asked $(n=6)$. The final sample size was 834 potential respondents.

\section{Statistical Methods}

The Quality of Life data (QoL) was first analyzed univariately by the Cochran-Mantel-Haenszel method for testing the repeated ordinal responses. 
Ranganathan Natarajan, et al. "Quality of Life in Chronic Kidney Patients Using a Synbiotic Dietary Supplement: a Survey"

For the multivariate analysis, the PROC GENMOD procedure in SAS was employed also due to the ordinal nature of the responses (specifically, the GEE method - Generalized Estimating Equations). This multinomial model allowed us to test whether the patient's QoL changed over time and also allowed us to correct/test the significance of other factors such as patient age, gender, and GFR. The model generates $\mathrm{p}$ values for each variable included in the model as well as odds ratios along with $95 \%$ confidence intervals.

This is a robust repeated-measures analysis. The GENMOD procedure (SAS) uses generalized estimating equations to account for non-independent data collected over time. Due to the fact that repeated measurements within patients may be correlated, this procedure allows one to model a "correlation structure" of the repeated measurements, commonly referred to as a covariance pattern. This accurate estimate will allow for improved estimates of the standard errors of measurement, and therefore more powerful tests. The exchangeable structure provides the best fit.

$\mathrm{P}$ values $<0.05$ were considered statistically significant but were not adjusted for multiple comparisons and any inflation of the type I error. Data were analysed using SAS system software (SAS Institute Inc., Cary, NC).

\section{RESULTS}

Of 834 potential respondents, $\mathrm{n}=168$ returned their questionnaires, a response rate of $20 \%$. Subsequently, $21 \%$ of the respondents $(n=36)$ claimed the offered discount. All results are reported below, and the percentages refer to a fraction of the total number of respondents $(n=168)$, unless otherwise indicated.

\section{A. Demographics}

The demographics of the sample population are presented in Figure 1. Over four fifths $(n=143,85 \%)$ of the respondents were older than 51 years, almost three quarters $(n=121,72 \%)$ - older than 61 years, and over a third $(n=65,39 \%)$ - older than 71years. Males $(n=79,47 \%)$ slightly outnumbered females $(n=71,42 \%)$. Almost one half $(n=80,48 \%)$ of the respondents were retired, another third $(n=51,30 \%)$ - employed or self-employed.

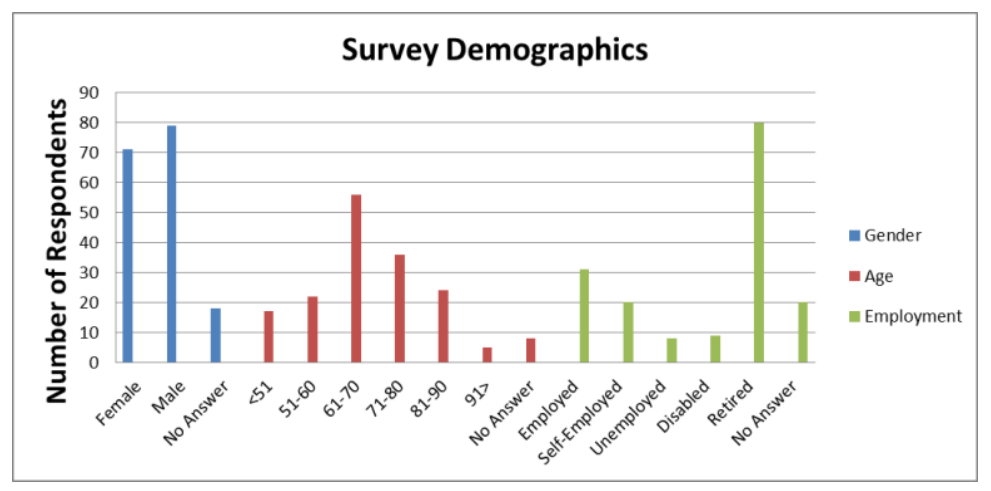

Figure1. Survey Demographics, $n(\%),(n=168)$

\section{B. Epidemiology}

The epidemiological characteristics of the study population are presented in Table 1 . The majority of respondents $(\mathrm{n}=118,70 \%)$ were diagnosed with $\mathrm{CKD}$ after turning 51 years old. Just over half $(\mathrm{n}=93$, $55 \%)$ were diagnosed when they were already in Stage 3 or 4 of the disease, while a quarter $(n=42$, $25 \%$ ) - when they were still in Stages 1 or 2. In addition, $58 \%$ of the respondents ( $n=97)$ reported being in Stages 3 or 4 of CKD at the time of their last doctor's visit, while another $17 \%(n=29)-$ in Stage 5 . The majority $(57 \%, \mathrm{n}=96)$ had their last doctor's visit sometime between July and December of 2015. Please see the Discussion section for a detailed analysis of these results.

When asked about the cause of their CKD, the respondents most frequently cited hypertension (17\%, $\mathrm{n}=28)$, an unknown cause $(15 \%, \mathrm{n}=25)$, diabetes $(14 \%, \mathrm{n}=23)$, and Polycystic Kidney Disease (PKD) $(8 \%, \mathrm{n}=13)$. In addition, a variety of other urinary system problems $(10 \%, \mathrm{n}=17)$, medication-related causes $(11 \%, \mathrm{n}=19)$, as well as immune (6\%), hereditary (5\%), age-related (4\%), cancer-related (4\%), surgery-related (3\%), or other cardiovascular causes $(2 \%)$ were cited. 
Ranganathan Natarajan, et al. "Quality of Life in Chronic Kidney Patients Using a Synbiotic Dietary Supplement: a Survey"

More than a third $(38 \%, n=64)$ of the respondents reported being anaemic, with half of those $(n=30)$ receiving either erythropoietin or other anaemia treatments. Over a third $(35 \%, n=61)$ had been advised about the need for dialysis or kidney transplant treatment or had already received/started treatment, which is approximately consistent with the proportion of the respondents being in Stages 4 (severe) and 5 (ESRD) of CKD $(41 \%, n=68)$ at the time of their last doctor's visit.

Table1. Survey Epidemiology, $n(\%),(n=168)$

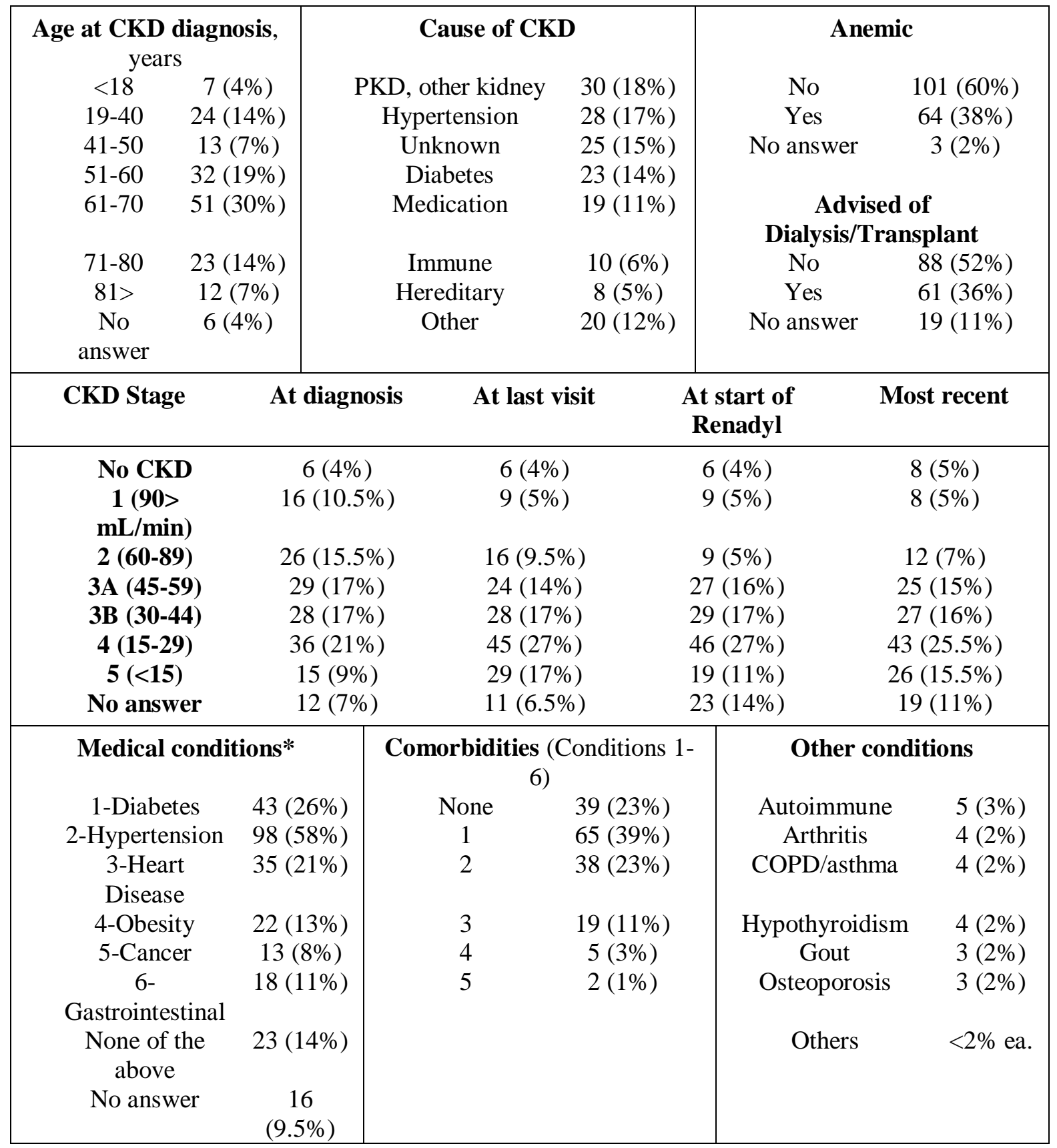

*Percentages do not add up to $100 \%$ due to comorbidities

Unsurprisingly, the majority of CKD patients suffered from either hypertension (58\%) or diabetes (26\%), or both simultaneously (17\%), the two leading risk factors for CKD. Moreover, $13 \%$ suffered from obesity, a significant risk factor for both diabetes and hypertension (indeed, $86 \%$ of respondents suffering from obesity also suffered from either or both conditions). Heart disease was a problem for $21 \%$, while cancer - for almost $8 \%$ of the respondents, with $77 \%$ of the cases in both instances comorbid with hypertension and/or diabetes. Just over $10 \%$ suffered from some type of gastrointestinal (GI) disorder, with $65 \%$ of these cases comorbid with hypertension and/or diabetes. Close to $9 \%$ of the respondents did not suffer from any additional medical conditions. Overall, given the six listed conditions (diabetes, hypertension, heart disease, obesity, cancer, and gastrointestinal 
Ranganathan Natarajan, et al. "Quality of Life in Chronic Kidney Patients Using a Synbiotic Dietary Supplement: a Survey"

disorders) more than a third $(39 \%, \mathrm{n}=65)$ had at least one of these conditions, almost a quarter $(23 \%$, $\mathrm{n}=38)$ - two comorbid conditions, a tenth $(11 \%, \mathrm{n}=19)$ - three conditions, and only a few $(4 \%, \mathrm{n}=7)-$ four or five comorbidities.

Some of the other conditions reported by the respondents included endocrine or metabolic disorders (5\%, gout, hypercholesterolemia, hypothyroidism), a variety of autoimmune disorders (5\%, allergies, lupus, vasculitis, eczema, etc.), skeletal/joint problems (5\%, osteoarthritis, osteoporosis), respiratory conditions (4\%, COPD, asthma, idiopathic pulmonary fibrosis), and mental or neurological disorders (3\%, depression/anxiety, bipolar, neuropathy, fibromyalgia).

\section{Renadyl-Related Questions}

The overwhelming majority of the respondents indicated that they took Renadyl for CKD ( $\mathrm{n}=149$, $89 \%)$, while a few $(\mathrm{n}=5,3 \%)$ took it because of other kidney health-related conditions or as a general preventative measure (Table 2). Most of the respondents $(\mathrm{n}=131,78 \%)$ have been taking Renadyl for 1 to 5 years, following the instructions on the package $(n=129,77 \%)$ (Table 3$)$. The suggested dosage was two capsules per day, taken one in the morning and one in the evening with meals. Those reporting different regimes from the one provided in the instructions cited taking fewer capsules due to cost $(n=5)$, forgetting/missing doses $(n=4)$, or quantity of pills $(n=1)$, or taking more capsules to increase effectiveness $(n=4)$ (table 4$)$.

The majority ( $\mathrm{n}=102,61 \%)$ reported being in stages 3 or 4 of CKD when they started taking Renadyl, while another 11\% ( $\mathrm{n}=19)$ - in Stage 5(Table 1 heading-at the start of Renadyl). The most recent CKD stage was reported as stages 3 or 4 by $56 \%(n=93)$, as stage 5 - by $16 \%(n=26)$. Please see Table 1 heading-most recent and the Discussion section for a detailed analysis of these results.

Table2. Renadyl Related Questions, $n(\%),(n=168)$

\begin{tabular}{|l|l|l|l|l|}
\hline \multicolumn{5}{|l|}{ Do you take Renady ${ }^{\text {TM }}$ to treat chronic kidney disease? Yes or No } \\
\hline \multirow{2}{*}{ Answer } & \multirow{2}{*}{ Frequency } & \multirow{2}{*}{ Percent } & Cumulative & Cumulative \\
\cline { 3 - 5 } & & Frequency & Percent \\
\hline Yes & 149 & 90.3 & 149 & 90.3 \\
\hline No & 16 & 9.7 & 165 & 100 \\
\hline Frequency Missing $=3$ & \multicolumn{5}{l}{} \\
\hline
\end{tabular}

Table3. Renadyl Related Questions, $n(\%),(n=168)$

\begin{tabular}{|l|l|}
\hline \multirow{3}{*}{ Years of taking Renadyl } & $<1$ year-23(14\%) \\
\cline { 2 - 2 } & $1-5$ years-131(78\%) \\
\cline { 2 - 2 } & Missing-14(8.0\%) \\
\hline
\end{tabular}

Table4. Renadyl Related Questions, $n(\%),(n=168)$

\begin{tabular}{|l|l|l|l|l|}
\hline Do you take Renadyl as per the instructions provided with the Renadyl package? \\
\hline \multirow{2}{*}{ Answer } & \multirow{2}{*}{ Frequency } & \multirow{2}{*}{ Percent } & Cumulative & Cumulative \\
\cline { 3 - 5 } & & Frequency & Percent \\
\hline Yes & 129 & 80.63 & 129 & 80.63 \\
\hline No & 31 & 19.38 & 160 & 100 \\
\hline Frequency Missing $=8$ & \multicolumn{2}{l}{} \\
\hline
\end{tabular}

\section{Quality of Life and Impact of Renady ${ }^{\mathrm{TM}}$}

The majority $(\mathrm{n}=137,82 \%)$ reported their current wellness as being good to excellent, and only $2 \%$ $(n=4)$ - as poor (Table 5b). The corresponding responses for current energy were $72 \%(n=121)$ and $6 \%(\mathrm{n}=10)$ (Table $5 \mathrm{c})$. This may be compared to the respondents' wellness at diagnosis, with only $59 \%(n=99)$ responding "good" to "excellent", while $21.5 \%(n=36)$ - "poor" or "very poor" (Table $5 a)$. The overwhelming majority was able to accomplish daily (82\%) and quarterly (80\%) activities, while $14 \%$ and $15 \%$, respectively, were able to do so only sometimes (Table 6). A large majority $(61 \%)$ of the respondents reported that Renadyl ${ }^{\mathrm{TM}}$ has made at least some $(40 \%)$ or great $(24.84 \%)$ impact on their lives. Another $29.94 \%$ remained neutral. Only $5 \%(n=8)$ reported no impact whatsoever, while none reported any negative impact on their life while they were taking Renadyl (Table 7). 
Ranganathan Natarajan, et al. "Quality of Life in Chronic Kidney Patients Using a Synbiotic Dietary Supplement: a Survey"

Table5. Impact of Renadyl on Quality of Life

5a. How was your wellness when you were first diagnosed with kidney disease?

\begin{tabular}{|l|l|l|l|l|}
\hline \multirow{2}{*}{ Answer } & \multirow{2}{*}{ Frequency } & \multirow{2}{*}{ Percent } & Cumulative & Cumulative \\
\cline { 4 - 6 } & & Frequency & Percent \\
\hline Excellent & 12 & 7.5 & 12 & 7.5 \\
\hline Good & 34 & 21.25 & 46 & 28.75 \\
\hline Fair & 47 & 29.375 & 93 & 58.125 \\
\hline Poor & 25 & 15.625 & 118 & 73.75 \\
\hline Very Poor & 26 & 16.25 & 144 & 90 \\
\hline
\end{tabular}

Frequency Missing = 8

5b. How is your current wellness:

\begin{tabular}{|l|l|l|l|l|}
\multirow{2}{*}{ Answer } & \multirow{2}{*}{ Frequency } & \multirow{2}{*}{ Percent } & Cumulative & Cumulative \\
\cline { 3 - 5 } & & Frequency & Percent \\
\hline Excellent & 18 & 10.78 & 18 & 10.78 \\
\hline Gory good & 53 & 31.73 & 71 & 42.51 \\
\hline Fair & 70 & 41.92 & 141 & 84.43 \\
\hline Poor & 26 & 15.57 & 167 & 100 \\
\hline Frequency Missing $=\mathbf{5}$ & 0 & 167 & 100 \\
\hline 5c. How is your current energy? & \multicolumn{5}{|l}{} \\
\hline $\begin{array}{l}\text { Answer } \\
\text { Fxcellent }\end{array}$ & Frequency & Percent & Cumulative & Cumulative \\
\hline Very good & 12 & 7.19 & 12 & Percent \\
\hline Good & 61 & 24.55 & 53 & 7.19 \\
\hline Fair & 68 & 40.72 & 121 & 31.74 \\
\hline Poor & 36 & 21.56 & 157 & 72.46 \\
\hline Frequency Missing $=\mathbf{1}$ & 5.99 & 167 & 94.01 \\
\hline
\end{tabular}

Table6. Quality of Life

\begin{tabular}{|l|l|l|l|l|}
\hline Are you able to achieve your daily tasks or chores? \\
\hline \multirow{2}{*}{ Answer } & \multirow{2}{*}{ Frequency } & \multirow{2}{*}{ Percent } & Cumulative & Cumulative \\
\cline { 3 - 5 } & & 83.03 & 137 & Pequency \\
\hline Yes & 137 & 1.82 & 140 & 83.03 \\
\hline No & 3 & 14.55 & 164 & 84.85 \\
\hline Sometimes & 24 & 0.61 & 165 & 99.39 \\
\hline Never & 1 & \multicolumn{5}{l}{} \\
\hline Frequency Missing $=\mathbf{3}$ & \multicolumn{5}{|l|}{} \\
\hline Are you able to achieve your quarterly tasks or chores? & Cumulative & Cumulative \\
\hline \multirow{2}{*}{ Answer } & Frequency & Percent & Frequency & Percent \\
\hline Yes & 135 & 82.32 & 135 & 82.32 \\
\hline No & 2 & 1.22 & 137 & 83.54 \\
\hline Sometimes & 26 & 15.85 & 163 & 99.39 \\
\hline Never & 1 & 0.61 & 164 & 100 \\
\hline Frequency Missing $=\mathbf{4}$ &
\end{tabular}

Table7. Impact of Renadyl

\begin{tabular}{|l|l|l|l|l|}
\hline How has Renadyl impacted your life? Please circle: \\
\hline & \multirow{2}{*}{ Frequency } & \multirow{2}{*}{ Percent } & Cumulative & Cumulative \\
\cline { 4 - 6 } Answer & & Frequency & Percent \\
\hline Great improvement & 39 & 24.84 & 39 & 24.84 \\
\hline Some improvement & 63 & 40.13 & 102 & 64.97 \\
\hline Neutral & 47 & 29.94 & 149 & 94.9 \\
\hline No improvement & 8 & 5.1 & 157 & 100 \\
\hline Frequency Missing $=\mathbf{1 1}$
\end{tabular}


Ranganathan Natarajan, et al. "Quality of Life in Chronic Kidney Patients Using a Synbiotic Dietary Supplement: a Survey"

\section{E. Renadyl and Glomerular Filtration Rate}

As CKD progresses the GFR number (which is an indirect measurement of the kidney's ability to filter uremic toxins) decreases. The higher the stage of CKD of the patient the worse the kidney failure. Customers taking Renadyl saw an improvement in the GFR. 56 (40\%) of customers were at stage 3 of CKD before they started taking Renadyl. This number decreased to 41(28.87\%) once they started taking Renadyl on a daily basis (Table 8). This indicates that Renadyl can maintain and improve kidney health in CKD patients and help kidneys from getting worse.

Table8. Effect of Renadyl on GFR (CKD stage)

\begin{tabular}{|c|c|c|c|c|}
\hline \multicolumn{5}{|c|}{ What was your GFR or stage of kidney disease when you started taking Renadyl? } \\
\hline \multirow{2}{*}{ Answer } & \multirow{2}{*}{ Frequency } & \multirow{2}{*}{ Percent } & Cumulative & Cumulative \\
\hline & & & Frequency & Percent \\
\hline Normal/ no CKD & \multirow{2}{*}{$\frac{2}{9}$} & \multirow{2}{*}{$\begin{array}{l}1.43 \\
6.43\end{array}$} & 2 & 1.43 \\
\hline Stage $1 \quad$ GFR $>90$ & & & 11 & 7.86 \\
\hline Stage 2 GFR $60-89$ & 9 & 6.43 & 20 & 14.29 \\
\hline Stage 3A $\quad$ GFR 45-59 & 27 & 19.29 & 47 & 33.57 \\
\hline Stage 3B GFR 30-49 & 29 & 20.71 & 76 & 54.29 \\
\hline Stage 4 GFR $15-29$ & 46 & 32.86 & 122 & 87.14 \\
\hline Stage 5 (ESRD) GFR $<15$ & 18 & 12.86 & 140 & 100 \\
\hline \multicolumn{5}{|c|}{ Frequency Missing $=28$} \\
\hline \multicolumn{5}{|c|}{ What was most recent GFR OR Stage of kidney disease? } \\
\hline \multirow{2}{*}{ Answer } & \multirow{2}{*}{ Frequency } & \multirow{2}{*}{ Percent } & Cumulative & Cumulative \\
\hline & & & Frequency & Percent \\
\hline Normal/ no CKD & 1 & 0.7 & 1 & 0.7 \\
\hline Stage $1 \quad$ GFR $>90$ & 8 & 5.63 & 9 & 6.34 \\
\hline Stage 2 GFR $60-89$ & 13 & 9.15 & 22 & 15.49 \\
\hline Stage 3A GFR 45-59 & 25 & 17.61 & 47 & 33.1 \\
\hline Stage 3B $\quad$ GFR 30-49 & 26 & 18.31 & 73 & 51.41 \\
\hline Stage 4 GFR 15-29 & 43 & 30.28 & 116 & 81.69 \\
\hline Stage 5 (ESRD) GFR $<15$ & 26 & 18.31 & 142 & 100 \\
\hline \multicolumn{5}{|c|}{ Frequency Missing = 26} \\
\hline
\end{tabular}

\section{F. Statistical Analysis}

\section{Univariate Method -Cochran-Mantel-Haenszel statistics testing the Ordinal response}

There was a difference in the distribution of Quality of Life (QoL) responses when comparing respondents before and after taking Renadyl and the numeric values of QoL differed across time, demonstrating improvement in Qol over time.

Table9. Summary Statistics for TIME (Before Renadyl vs. After) by QOL

\begin{tabular}{|c|c|c|c|c|}
\hline \multicolumn{5}{|c|}{ Cochran-Mantel-Haenszel Statistics (Based on Table Scores) } \\
\hline Statistic & Alternative Hypothesis & DF & Value & P value \\
\hline 1 & Nonzero Correlation* & 1 & 19.6597 & $<.0001$ \\
\hline 2 & Row Mean Scores Differ & 1 & 19.6597 & $<.0001$ \\
\hline 3 & General Association & 5 & 35.3613 & $<.0001$ \\
\hline
\end{tabular}

Multivariate Method - GEE Modeling (Generalized Estimating Equations for Ordinal Data - The Proportional Odds Model)

The following factors were found to be significantly associated with an improved QoL: the actual taking of Renadyl (TIME), respondents employed fared better than those not employed, the number of other medical conditions or diseases a respondent was suffering from (comorbidities) affected the respondent's QoL adversely and GFR (the higher the value, the better). It is also important to note that the following were tested for and found not to be associated with improved QoL: age, gender, diabetes, anemia, duration on Renadyl. 
Ranganathan Natarajan, et al. "Quality of Life in Chronic Kidney Patients Using a Synbiotic Dietary Supplement: a Survey"

Table10. GEE model

\begin{tabular}{|l|l|l|l|}
\hline Variable & p value & ODDS & {$[\mathbf{9 5 \%}$ Confidence Intervals] } \\
\hline Time & $<0.0001$ & 2.22 & {$[1.51,3.27]$} \\
\hline Working & 0.0114 & 2.01 & {$[1.18,3.44]$} \\
\hline Comorbidities & 0.0115 & 2.23 & {$[1.92,2.66]$} \\
\hline GFR & 0.0014 & 1.25 & {$[1.09,1.40]$} \\
\hline
\end{tabular}

Table11. $p$ value for parameters not associated with Quality of Life

\begin{tabular}{|c|c|}
\hline Age & 0.85 \\
\hline Gender & 0.26 \\
\hline Diabetic & 0.47 \\
\hline Anemic & 0.21 \\
\hline Erythropoietin & 0.57 \\
\hline Dialysis & 0.99 \\
\hline
\end{tabular}

It was seen that the odds were 2.22 to 1 that a respondent was in an improved QoL category at time 2 versus time 1 (baselines measure). Similarly, the odds were 2.01 to 1 that a respondent currently working was in a higher QoL category versus an unemployed (including retired or disabled). For each additional comorbidity, the odds were 2.23 to 1 that a respondent's QoL category declined. Similarly, for each 15-unit increase in GFR, the odds were 1.25 to 1 that the patient's QoL improved.

\section{DISCUSSION}

Overall, the results can be described as overwhelmingly positive, reinforcing the findings of the 2013 customer satisfaction survey [29]. The participants' feedback conveys high level of satisfaction with the product formulation, its safety and perceived efficacy. The table below shows a comparison of results from our two surveys. The first one was done in 2013 and the present one in 2015 (Table 12).

Table12. Comparison of two surveys

\begin{tabular}{|l|l|l|}
\hline \multicolumn{1}{|c|}{ Parameters } & $\mathbf{2 0 1 3}$ & $\mathbf{2 0 1 5}$ \\
\hline Number of Responders & 147 & 168 \\
\hline ESRD CKD 5 & $25(17 \%)$ & $29(17 \%)$ \\
\hline CKD 3 and 4 & $84(57 \%)$ & $97(58 \%)$ \\
\hline Quality of life-Increased sense of wellbeing(Mood , energy, fitness) & $107(73 \%)$ & $121(72 \%)$ \\
\hline Improvement after taking Renadyl & $108(73 \%)$ & $120(72 \%)$ \\
\hline
\end{tabular}

The results also describe the expected significant variation in the health status of persons using Renady ${ }^{\mathrm{TM}}$, depending on the stage of kidney disease, number of comorbidities, age and other salient factors. Although it is generally deemed subjective health-related quality of life (QoL) is an important measure of how disease affects the lives of patients [22, 23, 24]. While it is known that dialysis patients have decreased QoL relative to healthy individuals, little is known about QoL of CKD patients in pre-dialysis stages 1-4, before renal replacement therapy [25]. Therefore, the current survey study contributes to the efforts to fill that particular gap in knowledge. Moreover, given the increasing evidence that probiotics have a significant role to play in improving QoL in a variety of conditions, such as, for instance, colorectal cancer [26], cystic fibrosis [27], or a variety of gastrointestinal, immune and metabolic conditions [28, 29], the authors wanted to continue building up the evidence base with regard to the effect of Renadyl ${ }^{\mathrm{TM}}$ on persons with CKD. That renal failure patients have pathogenic gut microbes is now evident from a large number of researches. Studies by Vaziri et al. [30] have shown that renal failure patients have an imbalanced gut microflora, while a recent review of the studies with pro- and prebiotics summarized the role of the gut microflora in uremia and CKD [31]. CKD patients suffer from gut dysbiosis which contributes to build up of uremic toxins formed by the pathogenic gut bacteria. This in turn leads to poor QoL in Renal failure patients. The gut microbiome is therefore an important factor to be addressed and restoring the gut balance can improve the QoL. In addition, recent studies indicate that metabolites such as phenols and indoles, which are also uremic toxins, come from colonic fermentation [32].Studies with Renadyl in dialysis patients [ 20 ] showed that there was a decrease in the gut derived toxin-indoxyl glucuronide, reduction in C-reactive protein and, patients experienced a better QoL. Secondly several of the survey customers on Renadyl reported an improvement in their eGFR (Table 8) which indicated Renadyl could stabilize kidney function and in turn impart a better QoL. 
To ensure the internal validity of the questionnaire, several controls were deliberately incorporated, such as rephrasing and repetition of questions. The resulting responses to such questions demonstrate close correspondence. In addition, data analysis itself provided additional corroboration in support of internal validity. For example, as indicated in the Results section above, the proportion of the respondents reporting having been advised about the need for dialysis or kidney transplant treatment or already receiving treatment was consistent with the proportion of the respondents currently in late stages of CKD ( 4 or 5 ). The correspondence between the responses about current medications and the epidemiological profile of the respondents (Table 1) added another dimension to internal validation

\section{A. Methodological Limitations}

Uncertainty about the external validity, or representativeness, was among the major limitations of the study. Inevitably, the sample used in this survey was selected according to convenience - all of the current customers of Kibow. This was not a truly random sample, because Kibow's customers represent a self-selected sample of kidney disease patients who already view alternative medicine, including dietary supplements and probiotics, either in a positive light or at least with suspended disbelief. In other words, there was no possibility to control for placebo effect. In comparison with the estimates based on the results of NHANES III, in the current sample CKD stages 4 and 5 are overrepresented, stage 3 relatively underrepresented, while stages 1 and 2 - significantly underrepresented [33]. This is understandable, however, as in stages 1 and 2 the signs and symptoms of CKD are either still absent or very mild, and thus undiagnosed.

In addition, all of the results were self-reported, which is a common limitation of survey methodology as far as the ability to ascertain the accuracy of such observations. At the same time, this can also be considered as one of survey methodology's strengths, since there is no other practically useful way to easily and unobtrusively capture individuals' perceptions and subjective experiences, which are important factors to consider in any therapeutic situation.

\section{B. Response Rate}

The response rate of $20 \%$ may seem low at first glance - depending on the context, survey response rates can reach 60 or $70 \%$ or higher. However, that usually requires several waves of reminders and additional significant efforts to increase the rate of response. In this particular case, only one wave of responses was collected with only one reminder sent electronically close to the end of the collection period, and only the responses received within the first 1.5 months were included in the analysis. There is some evidence to suggest that subsequent waves may have different characteristics and thus make the results actually less representative [34]

Age is a significant factor and has been shown in prior research to affect the response rate [35, 36]. Considering that the customer population is skewed toward the elderly, CKD being a chronic disease with an onset late in life, this also helps explain the relatively low response rate. Moreover, selfadministered survey questionnaires permit the respondents to examine the questions prior to making the decision about participation, thus influencing the latter due to negative emotions connected to the topic (i.e., fear of revealing personal information) or to perceived high burden of the questions (i.e., complicated reports of past behaviors, lookup of household records) and similar considerations [37].

In other words, low response rate was to be expected, given the unique demographic makeup of the CKD population. Besides, in recent years, the basic inferential paradigm of survey research, which assumes 100 response rates on a probability sample, has been challenged [37]. Survey designs seeking high response rates entail high costs, usually generated by repeated efforts to obtain access to sample units and to address any concerns of the sample persons [38]. This customer satisfaction survey was limited with regard to funding access and was conducted at a minimal cost.

\section{Non-Response Bias}

An important issue that is sometimes connected to the low response rate is the nonresponse bias. Low response rates are open to interpretation - the respondents may represent subgroups of the target population, some subgroups may have systematically failed to respond or responded at a lower rate, the results may be consequently biased to an unknown extent. Concern with bias is key if the survey content is differentially perceived by population subgroups and if the response rate is low [34, 37].

In this particular case, potential subgroups can be identified as pre-dialysis (CKD stages 1-4) vs. dialysis (CKD stage 5, usually). Most persons using Renadyl ${ }^{\mathrm{TM}}$ tend to be pre-dialysis patients, and so 
only less than a fifth of the respondents in this survey were in ESRD (end-stage renal disease, or CKD stage 5). While dialysis patients can also benefit from using the product, the very fact of receiving dialysis may be a factor affecting their willingness to use or ability to afford the product.

However, response rates alone are not good indicators of non-response bias. It is a well-developed finding in the survey methodological literature that response rates by themselves are poor indicators of non-response bias [39, 40]. The search for mechanisms that link nonresponse rates and nonresponse bias should focus on the level of individual measures and not on the level of the survey. To predict what survey estimates are most susceptible to nonresponse bias, we need to understand how each survey variable relates to causes of survey participation [33]. It is also important to understand non-respondents - often the reasons for not returning the questionnaire include one of the following and more: never received it, never got around to it, too busy, forgot it or mislaid it, completed but never mailed, came at a bad time (i.e., ill), thought received it by mistake, seemed too long, not interested, never answer surveys [40].

\section{Incentive}

The survey methodological literature offers a number of techniques to increase response rates, including, for example, pre-notification and incentives. However, none of these measures - including incentives - is reliably related to the magnitude of nonresponse differences [37]. The use of incentives has become fairly common, and there is agreement that incentives, both monetary and non-monetary, increase overall response rates. The dilemma for survey researchers, then, is not whether to offer an incentive, but what kind of incentive, at what value, and when in the survey process to offer it. Generally, non-monetary incentives are less effective than monetary ones, and prepaid incentives are more effective than those conditional upon participation [41, 42].

One of the reasons incentives may work is related to a norm of reciprocity, whereby the potential respondent feels obligated to respond or return the favor by completing the survey. The recipient of the incentive, having benefited, feels indebted to the giver. This obligation to return the favor is less contingent on the value of the benefit received, than on the ethical principle of helping those who have helped you. Viewed this way, an incentive valued not only for its perceived cash value, but also because it represents the thoughtfulness and genuine appreciation of the giver [41]

This manner of thinking applied in the current study: since the respondents were Kibow's active customers, a $25 \%$ discount on the next order of Renadyl $\mathrm{TM}^{\mathrm{TM}}$ was deemed an appropriate reward for taking the time to complete the questionnaire. As it were, only $21 \%$ of the respondents $(n=36)$ chose to take advantage of the discount

\section{E. Stages of CKD}

Four questions - asked the same type of question, asking the respondents to identify their stage of CKD at various time periods: at diagnosis, as they started taking Renadyl ${ }^{\mathrm{TM}}$ and at last visit/most recently. Please refer to Table 1 for the summary of responses to these four questions. To explain and clarify the several discrepancies in response results, a more detailed analysis was performed. One of the questions that was raised pertained to the number of respondents indicating stage 5 (ESRD) of CKD: 15 of the respondents indicated already being in stage 5 at diagnosis, while 29 indicated stage 5 as their current stage of CKD. In addition, 19 respondents indicated being in stage 5 at the start of Renadyl ${ }^{\mathrm{TM}}$ administration.

First of all, it is important to account for the number of missing responses to each question. To begin with, it is very likely that many respondents were unable to recall their stage of CKD at a particular point in time when they started taking Renadyl ${ }^{\mathrm{TM}}$, as opposed to when they were first diagnosed or to their current status. In addition, it is possible that the respondents may have gotten somewhat less diligent about responding to each question towards the end of the survey, or may have felt that they already responded to similar questions earlier in the survey and so ignored the later questions as redundant.

Further examination yielded additional useful information. A subset of data was isolated, which included 39 respondents indicating being in CKD stage 5 in any of the four questions under examination. These were compared and distributed into two broad categories: 13 "new" patients those that were not at stage 5 of CKD either at diagnosis or at the start of Renadyl, but did indicate stage 5 as their current status; and 26 "old" patients - those that were already at stage 5 either at 
Ranganathan Natarajan, et al. "Quality of Life in Chronic Kidney Patients Using a Synbiotic Dietary Supplement: a Survey"

diagnosis or at the start of Renadyl. Each of the categories was further subdivided into several groups. Among the 13 "new" stage 5 patients, 7 progressed from stage 4 , another 5 - progressed from stages 1-3 (2 of these 5 explicitly indicated genetic causes, which explains the rapid progression), while 1 case represented an individual that had progressed to stage 5, but then improved due to kidney transplantation. Among the 26 "old" stage 5 patients, 8 improved and were no longer in stage 5 at the time of the survey, while 18 remained in stage 5 (Table1).

In summary, while it is hardly surprising that in general the severity of CKD progresses with time, there is some indication that Renadyl ${ }^{\mathrm{TM}}$ is able to stabilize and slow down this progression. One of the likely mechanisms by which this formulation of probiotics and prebiotics may be producing such an effect is through alleviating the dysbiosis of the gut microbiome, thus relieving many deleterious effects on the person's health. This approach has great potential in addressing the deterioration of GFR in persons with CKD. Given these preliminary indications, an adequately powered randomized controlled trial is warranted to investigate correlation between Renadyl ${ }^{\mathrm{TM}}$ administration and the rate of decline in GFR, to obtain a more objective measure than self-reported quality of life.

\section{F. Ethical considerations}

This customer satisfaction survey study can be classified as "minimal risk" research, which, in the clinical setting, usually receives expedited review from the Institutional Review Board (IRB), for which some or all elements of informed consent may be waived or modified, and in which vulnerable subjects including healthy children, incapacitated persons and prisoners may be permitted to enroll, even if a particular study does not hold out any direct benefit to them [43].

\section{CONCLUSIONS}

Renadyl ${ }^{\mathrm{TM}}$ appears to provide at least some benefit in all stages of CKD and with a variety of comorbid conditions. It does not interfere with any other medical treatments, including dialysis. It appears to have a stabilizing effect on the overall health status and quality of life, maintaining or improving kidney health in particular. Further adequately powered randomized controlled study to investigate possible correlation between Renadyl ${ }^{\mathrm{TM}}$ and the rate of decline in GFR is recommended.

\section{CONFLICT OF INTEREST}

Dr. Ranganathan and Mrs. Ranganathan are employees and stockholders of Kibow Biotech, Inc. Mr. Pechenyak and Mrs. Vyas are also employees and hold minor stock options in Kibow Biotech, Inc. Dr. D'Silva and Dr. Weinberg are consultants with a small token honorarium payment for their time and valuable efforts on this paper.

\section{REFERENCES}

[1] CDC Final Data for 2013: Leading causes of death in the U.S. http://www.cdc.gov/nchs/fastats/leading-causes-of-death.htm.

[2] USRDS Annual Data Report 2015, Volume 1: CKD in the United States and Volume 2: ESRD in the United States. http://www.usrds.org/2015/view/Default.aspx.

[3] Couser WG, Remuzzi G, Mendis S, Tonelli M,2011, The contribution of chronic kidney disease to the global burden of major noncommunicable diseases, Kidney Int, 80(12), 1258-1270

[4] Hill NR, Fatoba ST, Oke JL, Hirst JA et al,2016, Global prevalence of chronic kidney disease- a systematic review and meta-analysis. Plos One, http://dx.doi.org/10.1371/journal.pone.0158765

[5] Schepers E, Glorieux G, Vanholder R, 2010, The gut: The forgotten organ in uremia?, Blood Purification, 29,130-6.

[6] Stenvinkel P, 2006, Inflammation in end-stage renal disease: The hidden enemy, Nephrology, 11, 36-41.

[7] Shah SV, Baliga R, Rajapurkar M and Fonseca VA, 2007, Oxidants in chronic kidney disease, J Am Soc Nephrol, 18, 16-28.

[8] Karamouzis I, Sarafidis PA, Karamouzis M, Iliadis S, Haidich AB et al, 2008, Increase in oxidative stress but not in antioxidant capacity with advancing stages of chronic kidney disease, Am J Nephrol, 28, 397-404.

[9] Anders HJ, Andersen K, and Stecher B, 2013, The intestinal microbiota, a leaky gut, and abnormal immunity in kidney disease, Kidney International, 83(6), 1010-16. 
Ranganathan Natarajan, et al. "Quality of Life in Chronic Kidney Patients Using a Synbiotic Dietary Supplement: a Survey"

[10] Vaziri ND, Wong J, Pahl M, Piceno YM, Yuan J et al,2012, Chronic kidney disease alters intestinal microbial flora, Kidney International,, 83(2), 308-15.

[11] http://clinicaltrials.gov/ct2/results?term=probiotics\&Search=Search.Theclinicaltrials.gov website is a registry and results database of publicly and privately supported clinical studies of human participants conducted around the world.

[12] Ranganathan N, Patel BP, Ranganathan P, Marczely J, Dheer R et a. ,2006, In vitro and in vivo assessment of intraintestinal bacteriotherapy in chronic kidney disease, ASAIO Journal,52, 70-9.

[13] Ranganathan N, Patel B, Ranganathan P, Marczely J, Dheer R et al ,2005, Probiotic amelioration of azotemia in $5 / 6^{\text {th }}$ nephrectomized Sprague-Dawley rats, The Scientific World Journal,5,652-60.

[14] Ranganathan N, Patel B, Ranganathan P et al, 2005, Probiotics reduces azotemia in Gottingen mini-pigs. Proceedings of the 3rd World Congress of Nephrology Poster Presentation, Singapore.

[15] Palmquist R,2006, A Preliminary Clinical Evaluation of KibowBiotics®, a Probiotic Agent, on Feline Azotemia, Journal of the American Holistic Medical Association, 24(4), 23-7.

[16] Ranganathan N, Friedman EA, Tam P, Rao V, Ranganathan P et al, 2009, Probiotic dietary supplementation in patients with stage 3 and 4 chronic kidney disease: a 6-month pilot scale trial in Canada, Current Medical Research and Opinion, 25(8), 1919-30.

[17] Ranganathan N, Ranganathan P, Friedman EA, Joseph A, Delano B et al ,2010, Pilot Study of Probiotic Dietary Supplementation for Promoting Healthy Kidney Function in Patients with Chronic Kidney Disease, Advances in Therapy, 27(9), 634-47.

[18] Vitetta L, Gobe G2013, Uremia and Chronic Kidney Disease: The Role of the Gut Microflora and Therapies with Pro- and Prebiotics, Mol Nutr Food Res, 57, 824-32.

[19] Ranganathan N, Pechenyak B, Vyas U et al ,2013,Dose Escalation, Safety and Impact of a Strain-Specific Probiotic (Renadyl ${ }^{\mathrm{TM}}$ ) on Stages III and IV Chronic Kidney Disease Patients, Journal of Nephrology \& Therapeutics, 3,141. DOI: 10.4172/2161-0959.1000141.

[20] Ranganathan N, Pechenyak B, Vyas U et al 2014,Randomized Controlled Trial of Strain-Specific Probiotic Formulation (RENADYL ${ }^{\text {TM}}$ ) in Dialysis Patients, BioMed Research International,Volume 2014, Article ID 568571, 9 pages http;// dx.doi.org/10.1155/2014/568571

[21] Ranganathan N, Pechenyak B, Vyas U, Ranganathan P, DeLoach S et al ,2014,Review of Health Status and Level of Satisfaction of Customers with CKD Using Renadyl ${ }^{\mathrm{TM}}$ : Results of a Survey, International Journal of Medical and Applied Sciences, 3(3), 183-205.

[22] Patten, ML, 2001, Questionnaire Research: A Practical Guide, 2nd ed. Los Angeles: Pyrczak.

[23] Stewart AL, Hays RD, Ware JE, 1988, The MOS Short-Form General Health Survey: Reliability and Validity in a Patient Population, Medical Care, 26(7), 724-35.

[24] Carr-Hill RA, 1992, The Measurement of Patient Satisfaction, J Public Health Med, 14(3), 23649.

[25] Perlman RL, Finkelstein FO, Liu L, Roys E, Kiser M et al. Quality of Life in Chronic Kidney Disease (CKD): A Cross-Sectional Analysis in the Renal Research Institute - CKD Study. Am J Kidney Dis. 2005; 45(4): 658-66.

[26] Ohigashi S, Hoshino Y, Ohde S, Onodera H,2001, Functional outcome, quality of life, and efficacy of probiotics in postoperative patients with colorectal cancer, Surg Today, 41(9),1200-6.

[27] Jafari S-A, Mehdizadeh-Hakkak A, Kianifar H-R, Hebrani P, Ahanchain H et al 2013,Effects of Probiotics on Quality of Life in Children with Cystic Fybrosis; A Randomized Controlled Trial, Iran J Pediatr,23(6), 669-74.

[28] Ouwehand AC, Salminen S, Isolauri E, 2002, Probiotics: An Overview of Beneficial Effects, Antonie van Leeuwenhoek, 82, 279-89.

[29] Vyas U and Ranganathan N, 2012, Probiotics, Prebiotics, and Synbiotics: Gut and Beyond, Gastroenterol Res Prac, Article ID 872716, 16 pages. DOI: 10.1155/2012/872716

[30] N. D. Vaziri, J. Wong, M. Pahl, Piceno YM, Yuan J et al,2013, Chronic kidney disease alters intestinal microbial flora, Kidney International, 83(2),308-315

[31] L. Vitetta and G. Gobe,2013 Uremia and chronic kidney disease: the role of the gut microflora and therapies with pro-and prebiotics, Molecular Nutrition and Food Research, 57(5), 824-32. 
Ranganathan Natarajan, et al. "Quality of Life in Chronic Kidney Patients Using a Synbiotic Dietary Supplement: a Survey"

[32] P. A. Aronov, F. J. Luo, N. S. Plummer, Quan Z, Holmes S et al ,2011, Colonic contribution to uremic solutes, J Am Soc Nephrol, 22(9),1769-1776.

[33] Coresh J, Astor BC, Greene T, Eknoyan G, Levey AS, 2003, Prevalence of Chronic Kidney Disease and Decreased Kidney Function in the Adult U.S. Population: Third National Health and Nutrition Examination Survey, Am J Kidney Dis, 41(1), 1-12.

[34] Green KE, 1996, Sociodemographic Factors and Mail Survey Response, Psychology \& Marketing, 13(2), 171-84.

[35] Kaldenberg, D. O., Koenig, H. F., \& Becker, B. W,1994, Mail survey response rate patterns in a population of the elderly: Does response deteriorate with age?, Public Opinion Quarterly,, 58, 68-76.

[36] Herzog AR and Rodgers WL, 1988, Age and Response Rates to Interview Sample Surveys, Journal of Gerontology: SOCIAL SCIENCES, 43(6), S200-5.

[37] Groves RM, Peytcheva E, 2008, the Impact of Nonresponse Rates on Nonresponse Bias: A MetaAnalysis, Public Opinion Quarterly, 72(2), 167-89.

[38] Newby R, Watson J, Woodliff D,2003, SME Survey Methodology: Response Rates, Data Quality, and Cost Effectiveness, Entrepreneurship Theory and Practice, Winter issue, 163-72.

[39] Schouten B, Cobben F, Bethlehem J, 2009, Indicators for the representativeness of survey response, Survey Methodology, 35(1), 101-113.

[40] Rogelberg SG and Luong A, 1998, Nonresponse to Mailed Surveys: A Review and Guide, Curr Dir Psychol Sci, 7(2), 60-5.

[41] Ryu E, Couper MP, Marans RW, 2005, Survey Incentives: Cash vs. In-Kind; Face-to-Face vs. Mail; Response Rate vs. Nonresponse Error, International Journal of Public Opinion Research, 18(1), 89-106.

[42] Church A, 1993, Estimating the Effect of Incentives on Mail Survey Response Rates: A MetaAnalysis, Public Opinion Quarterly, 57, 62-79.

[43] Kopelman LM, 2004, Minimal Risk as an International Ethical Standard in Research, Journal of Medicine and Philosophy, 29(3), 351-78.

\section{AUTHOR'S BIOGRAPHY}

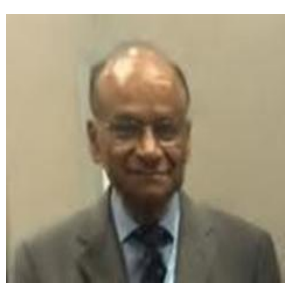

Dr. Rangan, is the key founder and Managing Director of Kibow Biotech, Inc a pioneering company in the field of understanding the Gut Microbiome, Dysbiosis, and its stabilization with Probiotics and Prebiotics towards health, disease and healing processes. As a medical R\&D professional, Dr. Rangan brings significant professional expertise and knowledge related to various medical specialties specifically - more so towards Chronic Kidney disease (CKD). He has dual master's degrees (organic chemistry and biochemistry) from the University of Poona, India and ad his doctoral degree in Bio-organic chemistry from Temple University, Philadelphia. He received further academic training at the University of Pennsylvania, Hahnemann Medical College, Philadelphia, and Johns Hopkins Medical Institutions, Baltimore. Dr. Rangan holds 24 patents and has several publications in peer reviewed nephrology and other medical journal. He is also a sought after speaker and reviewer in his field.

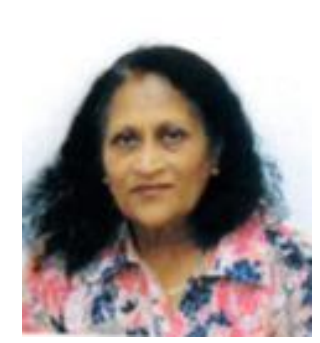

Pari Ranganathan, MS MT (ASCP) is V.P. of Clinical and Regulatory affairs at Kibow Biotech Inc for the last 16 years. She received her Bachelor of Science Degree in Chemistry from Women's College, Kerala, India. She then pursued her graduate education and obtained dual Master degree in Clinical Chemistry and Medical Technology from the University of Pennsylvania. Her initial work experience for 7 years was in the Clinical Laboratories at Presbyterian Medical Center of the University of Pennsylvania in Hematology, Coagulation and Hematology- Oncology. Subsequently she gained additional 20 years of experience in varied Management positions and finally advancing to the position of director of Clinical Laboratories in Presbyterian Penn Medicine - Univ of PA. Pari has been an associate member of the American Society of Clinical Pathology for the last 45 years. She has also worked as an Adjunct Faculty in Clinical Chemistry at Neumann University, PA. 
Ranganathan Natarajan, et al. "Quality of Life in Chronic Kidney Patients Using a Synbiotic Dietary Supplement: a Survey"

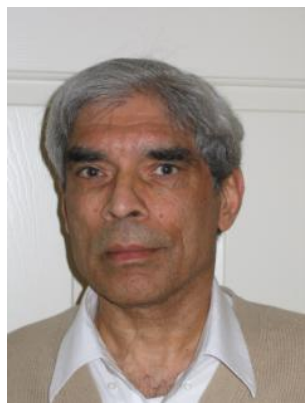

Dr Henry D'Silva, MD is a board certified nephrologist. He obtained his medical degree from Mumbai, India and completed his residency in Internal Medicine and fellowship in Nephrology from Thomas Jefferson University, Philadelphia, PA, USA. He is also certified in Medical Coding and billing by the American Association of Professional Coders (AAPC and CPC-A). He practised as a nephrologist from 1980 to 2002 at various hospitals in Albany and Troy in the state of New York and at hospitals in Lebanon and Hershey in Pennsylvania. Since 1997 he has been on Kibow Biotech's scientific advisory board giving valuable inputs in the development of Renadyl ${ }^{\mathrm{TM}}$ for kidney health. He has coauthored a book titled "Probiotics and Kidney health". In 2015 Dr Henry joined Kibow as a medical director for all of Kibow's products studied in human clinical trials.

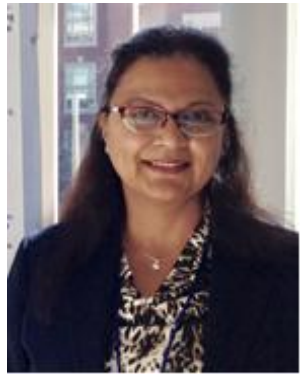

Usha Vyas, is a senior microbiologist at Kibow Biotech Inc., which make probiotic dietary supplement for Renal Health. She has a Bachelor's degree in Chemistry and a Master's degree in Microbiology from India. She worked for 22 years at Alembic Limited, a generic pharmaceutical company in India in their microbial fermentation research labs starting as a research scientist to Manager and heading the fermentation Research and Development labs. Her present work at Kibow involves developing new probiotic formulations for various applications, quality control for current products and managing all the lab operations. Since the past 7 years she has also been delivering talks at various forums to Renal Dieticians and Nephrology nurses educating them about the concept and importance of probiotics in health and disease and the modulation of the gut microbiome.

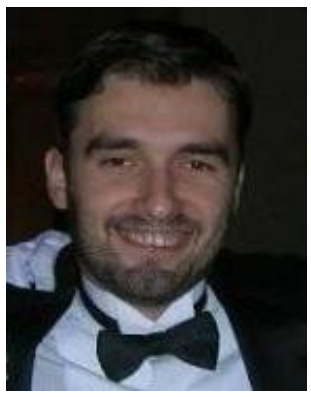

Bohdan Pechenyak, obtained his B.A. in sociology from Arcadia University in 2007 and his M.S.W. and M.P.H. from Temple University in 2011. Currently, he is a research specialist at Temple University School of Medicine.

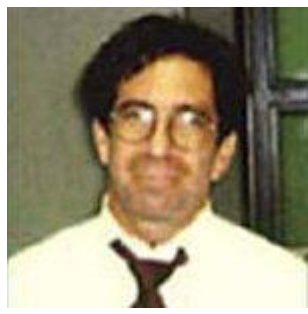

Alan Weinberg, has a Master's in Biostatistics from Columbia University, New York. He has been teaching mathematics and biostatistics actively at various universities including Seton Hall University from 1978 to 1992. From 19922008 he was a faculty member in the Departments of Surgery and Biostatistics at Columbia University. Presently he is an Associate Professor of Biostatistics in the Department of Population Health Science and Policy at the Mount Sinai School of Medicine. He continues to teach "Analytical Decision Making I" for the Executive MPH program at Columbia University. At Mt Sinai he teaches "Advanced Introduction to Biostatistics" and is the Course Director for "Computational Tools of Clinical Research." His statistical areas of expertise are in the analysis of clinical data, the design and analysis of experimental data, survival analysis and mixed modelling for the analysis of repeated measurements.

Citation: Ranganathan N, Ranganathan P, Henry D, Usha V, Bohdan P, Alan W. Quality of Life in Chronic Kidney Patients Using a Synbiotic Dietary Supplement: a Survey. International Journal of Research Studies in Medical and Health Sciences. 2017;2(1):11-24.

Copyright: ( 2017 Ranganathan N, Ranganathan P, Henry D, Usha V, Bohdan P, Alan W. This is an open-access article distributed under the terms of the Creative Commons Attribution License, which permits unrestricted use, distribution, and reproduction in any medium, provided the original author and source are credited. 